\title{
Low-cost Virtual Reality system in evaluation of rhythmic motor patterns in Elderly and Parkinsonian patients
}

\author{
Gabriel Sanmartín, Julián Flores \\ CoGRADE group(CITIUS) \\ Universidade de Santiago de Compostela, Spain \\ Verónica Robles-García, Pablo Arias, Javier Cudeiro \\ NEUROcom. Neuroscience and Motor Control Group \\ Universidade da Coruña, Spain
}

\begin{abstract}
This work details a Virtual Reality (VR) system developed to evaluate and potentially treat alterations in hand movements and central rhythm formation in Parkinsonian and Elderly subjects. An essential feature of VR systems to prove useful in clinical evaluation and to warrant "Presence", is the lack of behavioral distortion from real world execution.

Herein, we present a technical description of our VR and its validation to evaluate rhythmic motor patterns when experimental subjects perform a finger tapping test. Execution of the test was performed at different rates in the VR system, and compared to the gold-standard real world testing. The VR system proved to be as valid and reliable as real world testing to characterize arrythmokinetic profiles present in Parkinsonian and Elderly subjects (compared to Young subjects), at the different rates of execution. VR serves like a complementary tool in research settings to isolate subjects from un-naturalistic environment, during clinical evaluation, like lab-rooms or brain scans, since it did not bias behavior from real world evaluation in a basic clinical test.
\end{abstract}

\section{Introduction}

In the last few years, Virtual Reality (VR) has been extensively used and tested in multiple fields, with an increasing number of clinical studies on VR rehabilitation in motor pathologies such as stroke (Holden, 2001; Jack et al., 2001), brain damage (Rose, Brooks, \& Rizzo, 2005), phantom limb pain (Murray, Patchick, Pettifer, Caillette, \& Howard, 2006) or in treating Parkinson's Disease (PD) motor symptoms or non-motor symptoms, such

NEUROcom group, Universidade da Coruña.

Comments may be sent to the author at gabriel.sanmartin@usc.es 
as bradikinesia or gait impairments of executive dysfunction (Albani et al., 2002; Messier et al., 2007; Espay et al., 1998; Albani et al., 2010; Mirelman et al., 2010). The multiple benefits of VR over classic rehabilitation and training methods have been widely discussed (Holden \& Dyar, 2002; Rizzo \& Kim, 2005; Holden, 2005). By virtue of its flexibility, virtual environments can be easily modified and adapted to the need of each test, and also personalized for each subject according to their motor capabilities, motivation and goals. They can also provide immediate feedback in training setups, which enhances the learning process (Messier et al., 2007). Error-free learning has also been remarked as a means to improve learning by driving the user through visual or auditive cues that provide guidance towards successful error-free performance. Also, Virtual Environments (VE) incorporate a certain gaming factor that might help increasing motivation on the subject's by abstracting them from the usually distracting or intimidating clinical environments.

$\mathrm{PD}$ is a very common idiopathic neurodegenerative disorder, characterized by a spectrum of motor and non-motor symptoms (Jankovic, 2008). Motor symptoms include tremor, bradykinesia, rigidity and postural instability as cardinal signs, as exposed by Jankovic. A very common deficiency in Parkinsonian motor control is arrythmkinesia (instability in rhythmic movements in the temporal domain) which is also present in aging (Shimoyama, Ninchoji, \& Uemura, 1990). Arrythmkinesia is evaluated by means of the finger tapping test (Shimoyama et al., 1990) and reflects alteration in central control movement timing, which is also present in other more complex movements such as gait (Nakamura, Nagasaki, \& Narabayashi, 1976, 1978; Nagasaki et al., 1996; J. M. Hausdorff, Cudkowicz, Firtion, Wei, \& Goldberger, 1998; J. Hausdorff et al., 2003; Arias \& Cudeiro, 2008). It has been shown that patients improve their movements via an external cueing or guidance through specific stimuli (Rubinstein, Giladi, \& Hausdorff, 2002; Cunnington, Iansek, Bradshaw, \& Phillips, 1995; Morris, Iansek, Matyas, \& Summers, n.d.; Cunnington, Iansek, \& Bradshaw, 1999; Lewis, Byblow, \& Walt, 2000; Arias \& Cudeiro, 2010) which could be potentiated by the use of VR.

VR might also be very useful in clinical evaluation, for instance, by isolating subjects from un-naturalistic environments (scans, recording devices...), by creating more familiar surroundings, and by allowing configuration of specific experimental stimuli in a smartly controlled-way. However, to be useful, VR has to guarantee "Presence" which might be smartly evaluated by checking if execution in the VR is not different from execution in the real world. In other words, VR system has to be proven in its capability to offer a reliable experience that would faithfully reproduce real-life results into the VE. This is a basic element to consider VR among the available resources for evaluation of motor control in physiological and pathological conditions. This condition strongly relies on the VR creating a proper sense of Presence into the user.

Presence is accepted as the main element in the human interaction with Virtual Reality environments. There has been a long discussion regarding the conceptual definition of the term "presence" (e.g. Lee (2004), Coelho, Tichon, Hine, Wallis, and Riva (2006) or Schuemie, Straaten, Krijn, and Mast (2001)), but most agree on the generalized idea of presence as the illusion of "being there", whether or not "there" exists in physical space (Biocca, 1997).

There have been several methods used to measure the level of presence of a VR system and there is a long debate over what is the best practice for presence assessment. Many 
VR projects rely on subjective measures like scaled questionnaires (Witmer \& Singer, 1998; Dinh, Walker, Hodges, Song, \& Kobayashi, 1999; Schubert, Regenbrecht, \& Friedmann, 2001), continuous measure (IJsselsteijn \& de Ridder, 1998), comparative tests (Slater, 2000) or any other methods that demand the subject to input in any way their personal impression on the immersiveness of the environment. However, it's been argued that these methods provide more of a personal opinion than an objective measure (M. Slater, 1999). These reports are also argued to be directly linked to personal aspects of the user instead of real and reliable introspective reports (Nisbett \& Wilson, 1977). Other studies present a straight jump into the rehabilitation process without first assessing the level of presence or the consistency of results between real and virtual environments, limiting their results to the training performance and its potential transfer to the real world.

Schuemie et al. (2001) and Coelho et al. (2006) distinguish between subjective measures (e.g. questionnaires) and objective measures, like behavioral/performance measures or physiological measures. We have focused in "behavioral" presence as defined by Schuemie et al. (2001): "a level of presence that causes people to respond to mediated stimuli as if it were unmediated".

The coupling between execution in the virtual and real environments have been previously advanced (Arias, Robles-Garcia, Sanmartin, Flores, \& Cudeiro, 2012), where pathological (PD) and physiological subjects (elderly and young) show a pattern of execution that presents no differences as to the environment used (real vs. virtual). Remarkably, the task was executed at their Comfort rate, a tapping pattern considered as the "default" pattern, and likely the more "locked" within the motor repertoire of each subject. It is of great interest, therefore, to know if such coupling is maintained if the executed movement drifts off from the more "automatic pattern" where the role of feed-back from sensory resources (visual or somatic) may present a different gain. This is supported by the fact that changes in movement frequency present a differential brain activation dynamics; since the somatosensory cortex, the globus pallidus within the Basal Ganglia, and the cerebellum have been shown to be tuned to movement velocity (Turner, Grafton, Votaw, Delong, \& Hoffman, 1998) and these areas are directly related to sensorimotor integration, and in some cases are damaged in PD (Olanow \& Tatton, 1999). Keeping this in mind, the lack of differences in behavior between real and virtual environment at different movement frequencies would support the use of VR as a complementary tool for clinical evaluation and would also advocate its role as a rehabilitation tool in motor disorders.

In this paper we present an integral low-cost VR system using a HMD display and several motion capture techniques that reproduce a specific motor test that would allow experts and clinicians to evaluate physiological responses in the same way that they would in real environments. This, with the inherent advantages of VR systems, would then potentially prove useful to further evaluate patients through flexible environment and the potential treatment of patients with a future development of training exercises. We then proceed to evaluate the validity and reliability of the system by assessing its capability to detect alterations in the task, in three different groups of subjects, PD patients, elderly healthy controls and young healthy controls. Three different execution rates of tapping were performed (Turner et al., 1998), to further assess the capability of the VR system to reproduce alterations in the temporal domain associated with the task. 


\section{Experimental design}

The finger tapping test was used to evaluate subjects (Shimoyama et al., 1990). The test consists in tapping with the index finger (flexo-extension movements of the metacarpophalangeal joint) over a surface, it allows characterization of either hypokinetic and arrythmokinetic motor profiles.

The test was carried out as follows: the patient was sit on a chair, on a comfortable, relaxed position, with his hands and arms resting on a table and the elbow extended at about $90^{\circ}-100^{\circ}$. Subjects wore a Head-Mounted Display (HMD) which provides them with a first person view of a virtual environment specifically designed for the evaluation, representing the ideal environment for its execution: a square, empty room, except for a virtual depiction of himself (the "avatar"), the chair he is sit on and the work table.

The researcher requires then the subject to tap his finger, at different, comfortable rhythms: fast, normal and slow paces, for a number of times (see Methods for details). The movement of arms, hands and fingers is registered and reproduced in a realistic way, in real time, in the virtual environment, so the user perceives his tridimensional depiction as himself.

Our goal was to examine if execution in the VR environment is a valid method to detect impairment in rhythm formation in the different groups (validity), and if the execution in the VR can be reproduced under the same conditions as Real testing (reliability).

We included three different sets of subjects: Young healthy controls (YC), elderly healthy controls (EC), and Parkinson's disease (PD) patients. It has been shown that FT in the real world provides distinctive results in each of those groups (Arias, Robles-Garcia, Espinosa, Corral, \& Cudeiro, 2012).

The research hypothesis is that execution in the VR will reproduce execution in the real world in the different frequency-rates evaluated and in the three groups of subjects.

\section{Methods}

Subjects. All experimental subjects signed consent forms. The protocol conformed to the declaration of Helsinki and was approved by the Ethics Committee of the University of A Coruña (Spain) (CE-UDC 23/09-2009).

Young subjects 9 young healthy subjects (YH; based on medical history and personal interview) were recruited from staff and students of our institution (mean age 27.33 yrs; SD: 2.13).

Elderly controls 9 elder healthy subjects (EH based on medical history and personal interview) were recruited from relatives of staff working in our institution (mean age 66.22 yrs; SD: 2.95).

Parkinson's disease patients 10 idiopathic PD patients were recruited (mean age 67.10 yrs; SD: 2.92). Participants were recruited from our group database.

All participants were screened for dementia using MMSE (Folstein, Folstein, \& McHugh, 1975) and Edinburgh Handedness Inventory (EHI) (Oldfield, 1971). Subjects were 
excluded if they scored less than 24 in the MMSE or if they had any musculoskeletal impairment or disease apart from $\mathrm{PD}$ which might interfere with their ability to undertake the task. PD patients were evaluated OFF-periods (more than $12 \mathrm{~h}$ from last anti-parkinsonian medication intake, in the case the PD were taking slow-release antiparkinsonian medication at the end of the day, such intake was suppressed)

Procedure. Subjects performed the FT in the real world (FTREAL) and in the VR environment $\left(\mathrm{FT}_{\mathrm{VR}}\right)$. Both were performed by each subject at three different rates: Comfort; Slow-Comfort; and Fast-Comfort. Instructions given to the subjects were: "tap at your comfort rate" for Comfort; "tap not at your fastest rate but a fast rate which is comfortable and which you could maintain for a long time" for Fast-Comfort; and "tap at a slow but comfortable rate" for Slow-Comfort.

During the FT test subjects were comfortably sitting with forearms pronated on a table in front of them, so that both elbows were fixed at about $90-100^{\circ}$. Seat height was adapted so that subjects were in an optimal comfort position to perform the test. Participants performed the FT with their index finger by flexing-extending the metacarpophalangeal joint, and they were instructed to look at the hand executing the task. An event detector connected to a computer was attached at the tip of the index finger. Sampling rate was $1 \mathrm{KHz}$.

The same position was adopted by the avatar in the VR condition. The avatar was presented in 1st person perspective (egocentric perspective). This allowed the avatar's forearms to be perceived as if they were the subjects' own forearms. Before testing, VR environment was zoomed in and out to reach a natural avatar's hand size.

Subjects performed with their dominant hand (included dominance before diagnosis for the PD). Subjects were instructed to look at the executing hand, whether in the virtual or real environment. For VR, correcting lenses (Bobes, Inc, Madrid) were attached to each of the two screens of the HMD if necessary. Each rate was executed twice for each of the environments and tapping rates, during 50 cycles/each (apart from the first three discarded to allow rhythm stabilization). A rest period of $3 \mathrm{~min}$. was provided; and none of the subjects reported to feel fatigue throughout the protocol.

\section{Technical requirements}

As stated above, the intention of this study is to create a VR system such that for a specific task, the level of "Presence" is guaranteed to the point that no behavioral differences are induced by the VR environment (VE) compared to the real world. The design of such effective VE setup inevitably comes together with a series of requirements that must be satisfied to ensure this level of presence.

A motion capture system must be incorporated in order to allow the patient's natural interaction with the virtual environment. Other means of indirect interaction would not be valid for the purposes of this study, since they do not faithfully reflect the patient's real behavior and are obviously out of the scope of the intentions of this research. In particular, for the specific task chosen for this study (the finger-tapping test), a method for directly translating the movements as performed in the real environment would be needed. This means the whole transferring of finger, hand and arm motions. 
This motion capture solution and the virtual environment must be accurate enough so that suspension of disbelief is not broken. In other words, the environment and each of its elements (including the 3D representation of the user) must be realistic enough and reproduce real life behavior accurately in the tasks for which it has been designed. In the case of the environment this means that it should be designed based on reality and any sensory stimuli should be believable and according to the expectancies of the patient. Also responses induced in the neural system (motor control) should not be different from those induced by real sensory stimuli. For the motion capture system, a calibration process needs to be implemented and verified for the correct transferring of information to each of the tracked elements.

On the other hand, the end-to-end latency of the whole solution should be minimal. In other words, the delay between the moment the user performs an action and the time that action is shown on screen should be as low as possible; otherwise it would be induced a mismatch between somatosensory and visual systems information integration during the execution of the task, likely leading to sensorimotor distortion. Several studies have shown that high latency values have a negative effect on physiological presence (Meehan, Razzaque, Whitton, \& Brooks, 2003), and, in particular, on performance (Sheridan, 1992).

Lastly, since the system is intended for a future rehabilitation use by many users, we imposed the need of it meeting all the technical requirements while keeping the costs as low as possible.

\section{Hardware architecture}

Hardware architecture is a classical VR setup. It consists primarily of two computers following a server-client scheme, which conveniently parallels the expert-patient relationship during the tests. The "patient" computer is connected to the "expert" computer through an Ethernet network.

The "patient" computer centralizes most of the specific hardware, and controls most of the application logic: the 3D rendering, camera control and data acquisition. The second computer, ideally a laptop for greater flexibility, is used by the expert to control the exercise and test flow via specific software. Communication between all components is set through a conventional Ethernet LAN (Figure 1).

\section{Display devices}

Most VR setups for clinical applications revolve around three different display alternatives. The simplest of them is a regular computer monitor as in studies Cameirao, Badia, Oller, and Verschure (2010) or Merians, Poizner, Boian, Burdea, and Adamovich (2006). This option is the cheapest and easiest one, plus subjects tend to be used to its functioning, but it accounts for a lower sense of presence.

Other studies use CAVE-like setups (Keshner \& Kenyon, 2000; Whitney, Sparto, Furman, Jacobson, \& Redfern, 2002), which immerses the subject in a screened room surrounding the user, thus creating a whole immersive experience. This, though, requires expensive equipment and assembly.

A third option, perhaps the most common nowadays, is using a Head-MountedDisplay, which offer an immersive environment with a lower cost solution. In our case 


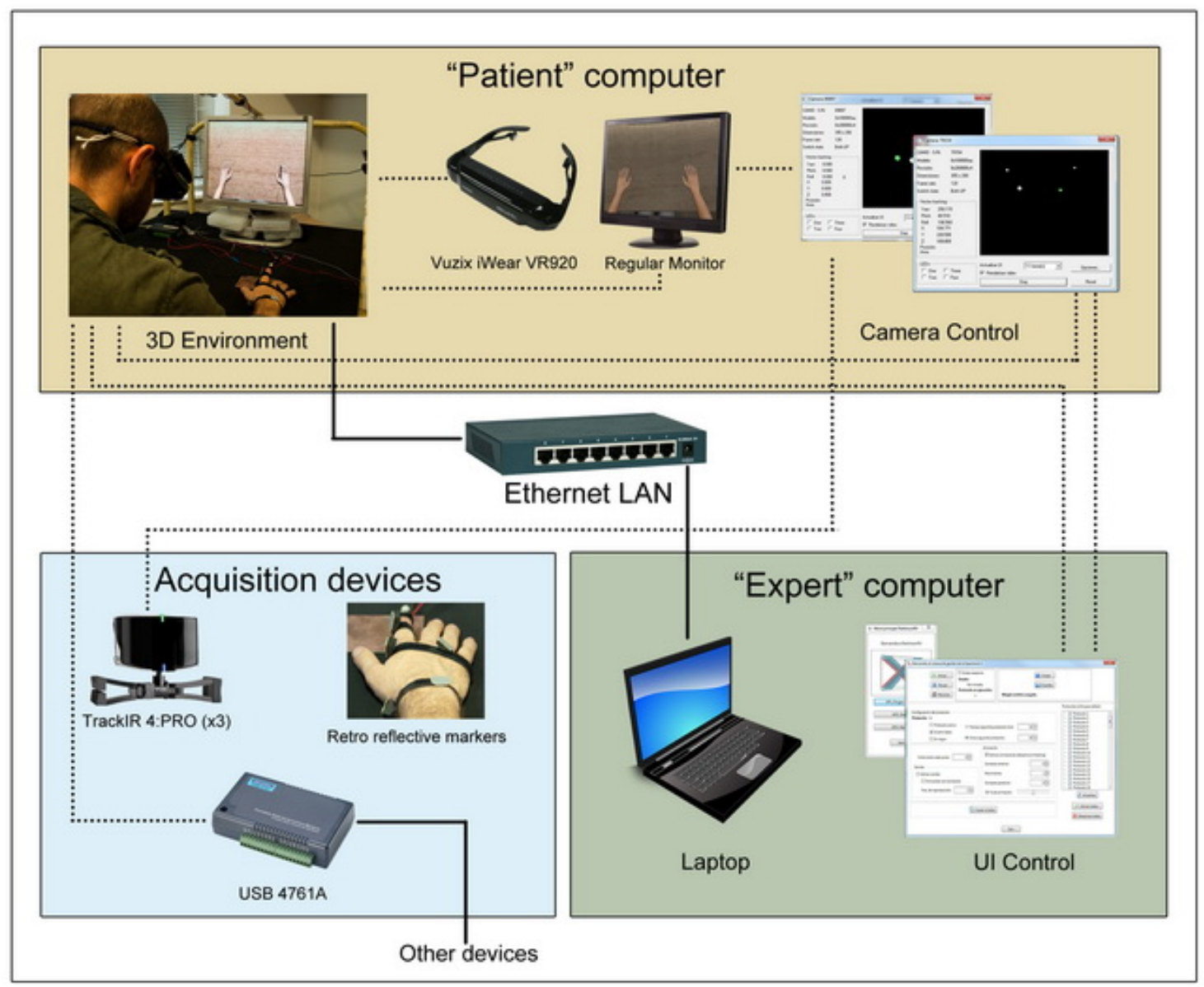

Figure 1. Scheme of the system: the main computer ("Patient") renders the Virtual Environment and camera control, while a second computer ("Expert") controls the test.

the patient wears a pair of immersive glasses with stereo capabilities for further immersion. Although these devices have been reported to increase the risk of cybersickness in the user, none of the control tests manifested any symptoms.

These devices also allow for an enhanced immersive experience compared to a regular display monitor, especially when head-tracking features are included. The model used for the project was a lightweight low-cost model, a pair of Vuzix iWear VR920 glasses (http://www.vuzix.com/home/) with full motion tracking and stereo imaging support, with a resolution up to $1024 \times 768$. Foam pads were used to further adjust the glasses and prevent sensory info from the real world.

An additional conventional display monitor is used in parallel in the patient computer, which reproduces the same image the user is viewing. This way the expert can also keep track of what the subject is experiencing and control the experience flow. Since the subject is wearing the HMD, this secondary monitor is not visible to him. 


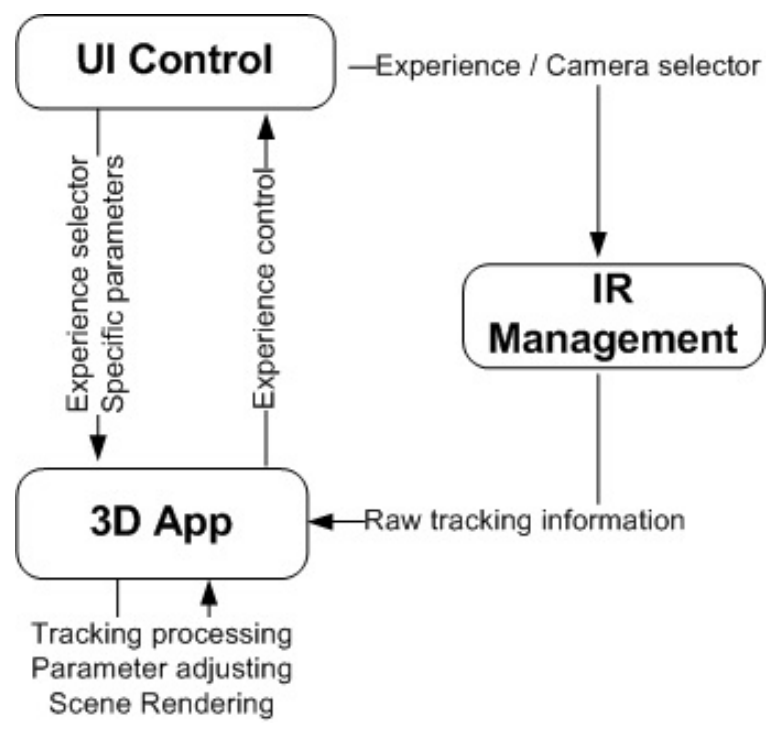

Figure 2. Communication diagram

\section{Tracking devices}

To allow interaction of the user in the virtual environment, a motion capture system specifically designed for this test was incorporated. We use optical tracking devices instead of other solutions, as they keep costs low while offering a fast, high frequency rate of capture.

Three infrared TrackIR4:PRO cameras from Natural Point are set up to capture the position and of several retro reflective markers. These cameras can work at $120 \mathrm{MHz}$ frequency and a resolution of 320x288 pixels each. Stability and spatial disposition of the cameras is granted by metallic supports designed by the research team.

\section{Software}

A modular set of applications was built for the Project, consisting of three separate elements (fig. 2). This design allows for substitution or scalability of the different modules independently while keeping the interoperatibility of the system.

The first module is in charge of 3D rendering and displaying the virtual environment, the application logic for the different tests, and the processing of the data received from the different sources (UI, tracking module, HMD and other devices...), as well as registering data.

A graphical user interface application was also built to allow supervision and full control over the course of the test, indicating both the 3D app and the Camera tracking module the specific parameters of the exercise. All settings can be altered in real-time, as data is constantly being streamed between the three modules.

A third module takes the role of handling the tracking devices, gathering the data obtained by them and adapt it to make it understandable by the $3 \mathrm{D}$ rendering program.

All three components were designed so by just meeting a certain interface any of them can be replaced without traumatic consequences. For example, the Camera Management 


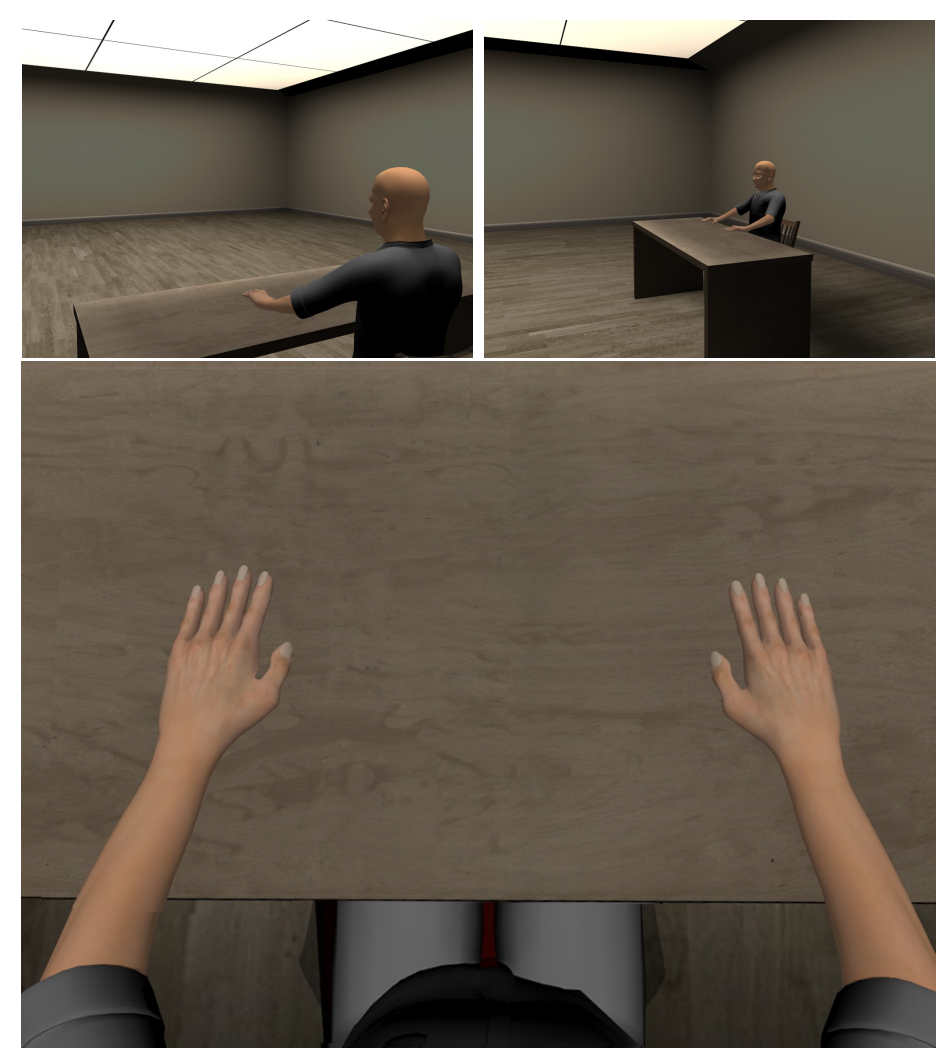

Figure 3. VR Environment

program could be replaced by another module controlling a totally different motion capture system and, by just forcing it to meet a certain interface, the system would still be working. This is, in fact, will allow further test development using the same UI and 3D programs.

\section{The Virtual Environment}

To ensure a sense of presence in the different tests, the environment must be as realistic as possible. To implement this realistic virtual environment we have chosen Ogre3D engine (http://www.ogre3d.org), as an open-source, scene-oriented, flexible 3D engine written in language $\mathrm{C}++$. We've proven over the years that Ogre3D is a reliable, fast and powerful engine that perfectly suits our needs.

A $3 \mathrm{D}$ scenario was modeled for its use in the tests, consisting of a big, square room in which a table and a chair are laid out, along with the virtual character. To prevent the patient from being visually distracted by other irrelevant objects for the experiment, the rest of the room is kept empty and aseptic. Brown and warm colors were applied to the scenery to dispel the cold sensations clinical environments usually produce. This proves as another advantage of VR testing as opposite to real life tests, as it is frequent for users or patients to get distracted or overwhelmed by clinical equipment and apparatus such as fMRI scans or other laboratory settings during the course of the tests.

The main element in the $3 \mathrm{D}$ rendering program is the virtual avatar. A generic human- 


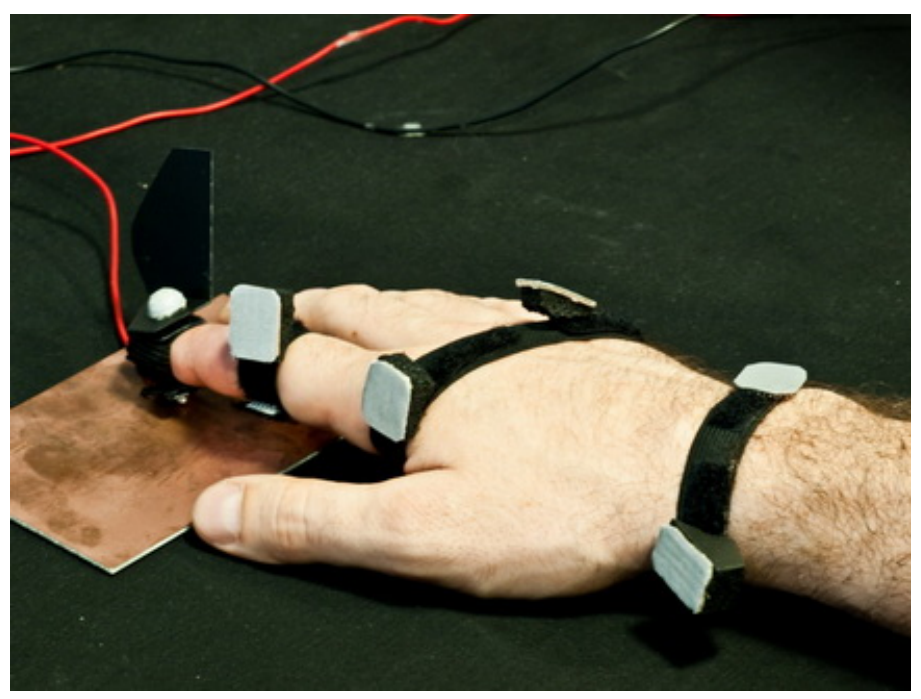

Figure 4. Layout of Retrorreflective markers

like 3D model was created using Maya and exporting to Ogre format, with a significant level of detail to ensure the realistic look on the scene, and ensuring suspension of disbelief on the patient.

This model was supplied with a rigging of animations which simulate the movements required for the patient, to offer the possibility for the expert to disable motion tracking and control the motion at will.

\section{Motion Tracking}

The tracking system provides rotational and positional information from a series of passive markers. This acquisition is done by a set of IR cameras described in the Hardware section. Markers are made out of adhesive markers of retro reflective material. Our software takes on the function of directly registering the positional information returned by IR cameras, whereas rotational information is obtained with the use of pairs of markers to get the value of angles for each of the three axes. Figure 4 shows the layout of the different markers.

A special case-based interpolation algorithm based on correspondence between successive frames was implemented to warrant temporal consistency of the markers and to enhance the stability of the system when marker occlusion or swapping occurs. Over the final result a Kalman filter (Jang, Kim, \& Choi, 1996) was applied to reduce vibrations caused by noise and to correct punctual errors in tracking.

No markers are needed for the arms, since the specific spatial position of the hand is enough to interpolate rotation and position of arm, forearm and shoulder thanks to the use of Inverse kinematics techniques. Specifically we have used a version of Cyclic-Coordinate Descent (Wang \& Chen, 1991). 


\section{Variables analyzed}

The variables analyzed were tapping frequency and the coefficient of variation $(\mathrm{CV})$ of inter-tap interval. The tapping frequency was calculated from the tapping events acquired from the event dectector (expressed in $\mathrm{Hz}$ ), which was also used to calculate the $\mathrm{CV}$ of inter-tap interval.

\section{Statistical analysis}

The statistical analysis included both tests of validity and reliability.

Validity. To evaluate the validity of $\mathrm{FT}_{\mathrm{VR}}$ for detecting differences in rhythm formation between groups an ANOVA with repeated measures was used. Factor Group (G) (between subjects factor) included three levels (each of the groups, PD, EC, YC).

Testing was performed at three different tapping rates, so a factor tapping-rate (TR) was defined with three levels (Comfort; Slow-comfort; Fast-comfort). Since the evaluation included Virtual and Real-world testing, a final factor (Condition; C) was defined, with two levels (Real and Virtual). Evaluation of main effect of factors and interaction between them allows understanding if groups' behavior was different in both environments and tapping rates, if ANOVA interactions involving group was not significant, subsequent analysis are run pooling groups.

Reliability. The reliability test compared the results obtained in VR and in the Real environments. We analyzed consistency in the execution between VR and Real-world by means of Intra-class Correlation Coefficient (ICC), for each variable. The mean difference between conditions was also analyzed, (Real minus Virtual for each subject), the closer to 0 the less different the execution.

Normality of distributions was assessed by means of one sample Kolmogorov-Smirnov test. When using ANOVA, univariate approaches were used to analyze within subject effects, for this the Greenhouse-Geisser coefficient was used in order to correct the degrees of freedom in case of sphericity violation.

Significance was set to 0.05. Results were presented as Mean and Standard of the Mean (SEM).

\section{Results}

Tapping Frequency. Validity and reliability of the VR system in the different tapping rates performed. Figure A1 in the appendix shows how the frequency of tapping was modulated at the three tapping rates $(\mathrm{p}<0.001)$. In all cases the test was able to characterize the groups, showing its validity to differentiate between them $(\mathrm{p}=0.006-\mathrm{PD}$ had higher frequencies than the other two groups at all tapping rates). Importantly this was observed in both conditions, VR and Real world $(\mathrm{p}=0.465)$, since no difference was observed between them; and this was not different depending on the group $(\mathrm{p}=0.623)$.

Since we did not observe a different behavior based on group factor, the subsequent reliability analysis was performed with the groups pooled, which shows (Fig. A2) a high level of consistency when comparing execution in the VR and Real world (ICC=0.98), with the mean difference between conditions not significantly different from 0 . 
$C V$ of inter-tap interval. Validity and reliability of the $V R$ system in the different tapping rates performed. If the analysis focuses on the CV the same outcomes as before (for frequency) can be observed. First, no difference was observed between Real and VR testing $(\mathrm{p}=0.135)$, and this was not different for the different groups in all tapping rates $(\mathrm{p}=0.928)$. The test was however able to characterize groups, showing a different behavior between them $(\mathrm{p}=0.003)$ (higher variability in $\mathrm{PD}$, then $\mathrm{HE}$, and the lowest for $\mathrm{YC}$ ), see Fig 3. In all groups variability seemed to be higher at slow tapping-rates $(p=0.056-a$ representative example for each group is shown in Fig. A4.

Again, as for the FQ, the reliability analysis showed high consistency when comparing execution in the virtual and real worlds ( $\mathrm{ICC}=0.89$; groups pooled); and also a mean difference between conditions at execution not significantly different from 0 was observed (Fig. A5).

\section{Discussion}

We introduced a VR system designed for clinical evaluation of repetitive finger movements in order to assess physiological alterations. Since the test reflects central rhythm formation, our results, showing that VR testing matches Real testing, support the use of VR in neurophysiological evaluations.

Remarkably, our results expand previous works on the suitability of VR to clinical testing (Arias, Robles-Garcia, Sanmartin, et al., 2012). Here we show VR suitability is not dependent on the frequency of movements executed by the subjects. This is important since there are reports of differential activation pattern in several sensorimotor processing nucleus, like the somatosensory cortex, the globus pallidus or the cerebellum (Turner et al., 1998). Even though some of these areas are impaired in the parkinsonian patients (globus pallidus belonging to the Basal Ganglia, as pointed out in Olanow and Tatton, 1999) the change in the features of execution in the patients is similar in the VR and Real environments. Also, cerebellum has a clear role in the control of the actual movement to the programmed movement (Kandel, Schwartz, \& Jessell, 2000), and it also revises somatosensory and visual input (Kandel et al., 2000) for which its role might be of critical importance in the case the VR systems creates a mismatch between visual and somatosensory information during the execution of the task which would drift execution in the VR away from that in the Real world, however this was not the case, advocanting the use of VR in neurophysiological and behavioural evaluation.

The induction of "Presence" is a basic feature in VE. The term "Presence" (see Introduction for conception) is hard to define and even harder to evaluate. There have been several approaches to evaluate the sense of Presence induced by different VR systems, some of them from a subjective point of view (e.g., Witmer \& Singer, 1998), which on the other hand has been criticized (M. Slater, 1999), and the use of objective measures has been encouraged (Schuemie et al., 2001; Coelho et al., 2006).

Here we proposed that we should match execution in VR to that in the Real world to guarantee a behavioral concept of presence. Both executions were objectively evaluated by means of a worldwide and valid motor test, useful in clinical practice and research the finger tapping test. In all motor patterns, resulting VR tests matched Real world in terms of motor behavior. This is of importance because, even if matching VR and Real world had been previously shown (Arias, Robles-Garcia, Sanmartin, et al., 2012), int that 
case the execution was done at the "default" comfort pattern, which becomes the automatic execution for each subject. In this case, by showing VR and Real execution at different frequencies (which makes them less automatic) and with a different brain activation profile (Turner et al., 1998), the suitability of the test in terms of "Presence" is reinforced.

For instance, the cerebellum has a deep role in motor control for comparing the current movement with the intended movement, but it immediately receives and integrates such information with sensory input from visual and somatic systems, so that any kind of mismatch between current movements and motion capture and reproduction would have, probably, an impact on motor execution. Since this was not the case, this strengthens the sense of Presence and the suitability of the system for the proposed objective.

\section{References}

Albani, G., Pignatti, R., Bertella, L., Priano, L., Semenza, C., Molinari, E., ... Mauro, A. (2002). Common daily activities in the virtual environment: a preliminary study in parkinsonian patients. Neurological Sciences, 23, s49-s50. Retrieved from http://dx.doi.org/10.1007/s100720200064 (10.1007/s100720200064)

Albani, G., Raspelli, S., Carelli, L., Morganti, F., Weiss, P., Kizony, R., ... Riva, F. (2010). Executive functions in a virtual world: a study in parkinson's disease. Stud Health Technol Inform, 154, 92-6. Retrieved from http://www. biomedsearch.com/nih/Executive-functions-in-virtual-world/20543277.html

Arias, P., \& Cudeiro, J. (2008). Effects of rhythmic sensory stimulation (auditory, visual) on gait in parkinson's disease patients. Experimental Brain Research, 186, 589-601. Retrieved from http://dx.doi.org/10.1007/s00221-007-1263-y doi: 10.1007/s00221-007-1263-y

Arias, P., \& Cudeiro, J. (2010, 03). Effect of rhythmic auditory stimulation on gait in parkinsonian patients with and without freezing of gait. PLOS ONE, 5 .

Arias, P., Robles-Garcia, V., Espinosa, N., Corral, Y., \& Cudeiro, J. (2012). Validity of the finger tapping test in parkinson's disease, elderly and young healthy subjects: Is there a role for central fatigue. Clinical neurophysiology : official journal of the International Federation of Clinical Neurophysiology, 123, 2034-41.

Arias, P., Robles-Garcia, V., Sanmartin, G., Flores, J., \& Cudeiro, J. (2012, 01). Virtual reality as a tool for evaluation of repetitive rhythmic movements in the elderly and parkinson's disease patients. PLoS ONE, 7, e30021. doi: 10.1371/journal.pone.0030021

Biocca, F. (1997). The cyborg's dilemma: Progressive embodiment in virtual environments. $J$. Computer-Mediated Communication, 3(2), 0.

Cameirao, M., Badia, B., Oller, E., \& Verschure, P. (2010). Neurorehabilitation using the virtual reality based rehabilitation gaming system: methodology, design, psychometrics, usability and validation. Journal of NeuroEngineering and Rehabilitation, 7, 1-14. Retrieved from http://dx.doi.org/10.1186/1743-0003-7-48 doi: 10.1186/1743-0003-7-48

Coelho, D. C., Tichon, P. J., Hine, D. T., Wallis, D. G., \& Riva, P. G. (2006). Media presence and inner presence: The sense of presence in virtual reality technologies. IOS Press, Amsterdam. Retrieved from http://cogprints.org/5965/ (Emerging Communication Series. Full book available online here: http://www.emergingcommunication.com/volume9.html)

Cunnington, R., Iansek, R., \& Bradshaw, J. (1999). Movement-related potentials in parkinson's disease: external cues and attentional strategies. Mov Disord, 14 (1), 63-8.

Cunnington, R., Iansek, R., Bradshaw, J. L., \& Phillips, J. G. (1995). Movementrelated potentials in parkinson's disease. presence and predictability of temporal and spatial cues. Brain, 118 ( Pt 4), 935-50. Retrieved from 
http://www.biomedsearch.com/nih/Movement-related-potentials-in-Parkinsons/7655889.html

Dinh, H., Walker, N., Hodges, L., Song, C., \& Kobayashi, A. (1999, mar). Evaluating the importance of multi-sensory input on memory and the sense of presence in virtual environments. In Virtual reality, 1999. proceedings., ieee (p. 222 -228). doi: 10.1109/VR.1999.756955

Espay, A. J., Baram, Y., Burton, N., Gartner, M., Miranda, H. A., Duker, A. P., \& Revilla, F. J. (1998). At-home training with virtual reality cues for improvement of gait in patients with parkinson's disease. Neurology, 11(70), A425-A425.

Folstein, M. F., Folstein, S. E., \& McHugh, P. R. (1975, November). "Mini-mental state". A practical method for grading the cognitive state of patients for the clinician. Journal of psychiatric research, 12(3), 189-198. Retrieved from http://view.ncbi.nlm.nih.gov/pubmed/1202204

Hausdorff, J., Schaafsma, J., Balash, Y., Bartels, A., Gurevich, T., \& Giladi, N. (2003). Impaired regulation of stride variability in parkinson's disease subjects with freezing of gait. Experimental Brain Research, 149, 187-194. Retrieved from http://dx.doi.org/10.1007/s00221-002-1354-8 doi: 10.1007/s00221-002-1354-8

Hausdorff, J. M., Cudkowicz, M. E., Firtion, R., Wei, J. Y., \& Goldberger, A. L. (1998). Gait variability and basal ganglia disorders: Stride-to-stride variations of gait cycle timing in parkinson's disease and Huntington's disease. Mov. Disord., 13(3), 428-437. Retrieved from http://dx.doi.org/10.1002/mds.870130310 doi: 10.1002/mds. 870130310

Holden, M. K. (2001). Neurorehabilitation using 'learinng by imitation' in virtual environments.

Holden, M. K. (2005). Virtual environments for motor rehabilitation: review. CYBERPSYCHOLOGY AND BEHAVIOR, 8(3), 187-211.

Holden, M. K., \& Dyar, T. (2002). Virtual environmnent training: A new tool for neurorehabilitation. Neurology Report, 26(2), 62-71.

IJsselsteijn, W. A., \& de Ridder, H. (1998). Measuring Temporal Variations in Presence.

Jack, D., Boian, R., Merians, A. S., Tremaine, M., Burdea, G. C., Adamovich, S. V., .. Poizner, H. (2001). Virtual reality-enhanced stroke rehabilitation. IEEE Lts, 9, 308-318. doi: $10.1109 / 7333.948460$

Jang, D.-S., Kim, G.-Y., \& Choi, H.-I. (1996, November). Kalman filter incorporated model updating for real-time tracking. In Tencon '96. proceedings. 1996 ieee tencon. digital signal processing applications (Vol. 2, p. 878 -882 vol.2). doi: 10.1109/TENCON.1996.608463

Jankovic, J. (2008). Parkinson's disease: clinical features and diagnosis. Journal of Neurology, Neurosurgery and Psychiatry, 79(4), 368-376. Retrieved from http://jnnp.bmj.com/content/79/4/368.abstract doi: 10.1136/jnnp.2007.131045

Kandel, E. R., Schwartz, J. H., \& Jessell, T. M. (2000, July 01). Principles of Neural Science. In (4th ed., p. 833-852). McGraw-Hill Medical. Hardcover. Retrieved from http://www. amazon.com/exec/obidos/redirect?tag=citeulike07-20\&path=ASIN/0071120009

Keshner, E. A., \& Kenyon, R. V. (2000, January 1). The influence of an immersive virtual environment on the segmental organization of postural stabilizing responses. Journal of Vestibular Research, 10(4), 207-219. Retrieved from http://iospress .metapress. com/content/G4BWW85N93NG57KV

Lee, K. M. (2004). Presence, explicated. Communication Theory, 14(1), 27-50. Retrieved from http://dx.doi.org/10.1111/j.1468-2885.2004.tb00302.x doi: 10.1111/j.14682885.2004.tb00302.x

Lewis, G. N., Byblow, W. D., \& Walt, S. E. (2000). Stride length regulation in parkinson's disease: the use of extrinsic, visual cues. Brain, 123(10), 2077-2090. Retrieved from http://brain.oxfordjournals.org/content/123/10/2077. abstract doi: 10.1093/brain/123.10.2077

Meehan, M., Razzaque, S., Whitton, M. C., \& Brooks, F. P., Jr. (2003). Effect of latency on presence in stressful virtual environments. In Proceedings of the ieee virtual re- 
ality 2003 (pp. 141-). Washington, DC, USA: IEEE Computer Society. Retrieved from http://dl.acm.org/citation. cfm?id=832289.835964

Merians, A. S., Poizner, H., Boian, R., Burdea, G., \& Adamovich, S. (2006). Sensorimotor Training in a Virtual Reality Environment: Does It Improve Functional Recovery Poststroke?

Messier, J., Adamovich, S., Jack, D., Hening, W., Sage, J., \& Poizner, H. (2007). Visuomotor learning in immersive 3d virtual reality in parkinson's disease and in aging. Experimental Brain Research, 179, 457-474. Retrieved from http://dx.doi.org/10.1007/s00221-006-0802-2 doi: 10.1007/s00221-006-0802-2

Mirelman, A., Maidan, I., Jacobs, A., Mirelman, D., Giladi, N., \& Hausdorff, J. (2010). 294 VIRTUAL REALITY FOR GAIT TRAINING IN PARKINSON'S DISEASE: A FEASIBILITY STUDY. Parkinsonism and Related Disorders, 16. doi: 10.1016/S1353-8020(10)70295-9

Morris, M. E., Iansek, R., Matyas, T. A., \& Summers, J. J. (n.d.).

Murray, C. D., Patchick, E., Pettifer, S., Caillette, F., \& Howard, T. (2006). Immersive virtual reality as a rehabilitative technology for phantom limb experience: A protocol.

Nagasaki, H., Itoh, H., K, H., T, F., H, M., \& T, K. (1996). Walking patterns and finger rhythm of older adults. Perceptual and motor skills, 82, 435-47.

Nakamura, R., Nagasaki, H., \& Narabayashi, H. (1976). Arrhythmokinesia in parkinsonism. Advances in Parkinsonism, 258-268.

Nakamura, R., Nagasaki, H., \& Narabayashi, H. (1978). Disturbances of rhythm formation in patients with parkinson's disease: part i. characteristics of tapping response to the periodic signals. Perceptual and motor skills, 46, 63-75.

Nisbett, R. E., \& Wilson, T. D. (1977, May). Telling More Than We Can Know: Verbal Reports on Mental Processes. Psychological Review, 84(3). Not available electronically.

Olanow, C. W., \& Tatton, W. G. (1999). ETIOLOGY AND PATHOGENESIS OF PARKINSON'S DISEASE. Annual Review of Neuroscience, 22, 123-144. doi: 10.1146/annurev.neuro.22.1.123

Oldfield, R. C. (1971, March). The assessment and analysis of handedness: The Edinburgh inventory. Neuropsychologia, 9(1), 97-113. Retrieved from http://dx.doi.org/10.1016/0028-3932(71)90067-4

Rizzo, A., \& Kim, G. J. (2005, April). A swot analysis of the field of virtual reality rehabilitation and therapy. Presence: Teleoper. Virtual Environ., 14(2), 119-146. Retrieved from http://dx.doi.org/10.1162/1054746053967094 doi: 10.1162/1054746053967094

Rose, F. D., Brooks, B. M., \& Rizzo, A. A. (2005, June). Virtual reality in brain damage rehabilitation: review. Cyberpsychology \& behavior: the impact of the Internet, multimedia and virtual reality on behavior and society, 8(3), 241-262. Retrieved from http://dx.doi.org/10.1089/cpb.2005.8.241 doi: 10.1089/cpb.2005.8.241

Rubinstein, T., Giladi, N., \& Hausdorff, J. (2002). The power of cueing to circumvent dopamine deficits: a review of physical therapy treatment of gait disturbances in parkinson's disease. Mov Disord, 17(6), 1148-60.

Schubert, T., Regenbrecht, H., \& Friedmann, F. (2001). The experience of presence: Factor analytic insights. presence: Teleoperators and virtual environments. In (pp. 266-281).

Schuemie, M. J., Straaten, P. V. D., Krijn, M., \& Mast, C. A. V. D. (2001). Research on presence in virtual reality: A survey. Cyberpsychology and Behavior, 4 (2), 183-201.

Sheridan, T. B. (1992). Musings on telepresence and virtual presence. Presence, 120-125.

Shimoyama, I., Ninchoji, T., \& Uemura, K. (1990). The finger-tapping test: A quantitative analysis. Archives of Neurology, 47(6), 681-684. Retrieved from + http://dx.doi.org/10.1001/archneur.1990.00530060095025 doi: 10.1001/archneur.1990.00530060095025

Slater. (2000). A virtual presence counter.

Slater, M. (1999, October). Measuring presence: A response to the witmer and singer pres- 
ence questionnaire. Presence: Teleoper. Virtual Environ., 8(5), 560-565. Retrieved from http://dx.doi.org/10.1162/105474699566477 doi: 10.1162/105474699566477

Turner, R. S., Grafton, S. T., Votaw, J. R., Delong, M. R., \& Hoffman, J. M. (1998). Motor Subcircuits Mediating the Control of Movement Velocity: A PET Study. Journal of Neurophysiology, 80(4), 2162-2176.

Wang, L.-C., \& Chen, C. (1991, August). A combined optimization method for solving the inverse kinematics problems of mechanical manipulators. Robotics and Automation, IEEE Transactions on, 7(4), 489 -499. doi: 10.1109/70.86079

Whitney, S., Sparto, P., Furman, J., Jacobson, J., \& Redfern, M. (2002). The potential use of virtual reality in vestibular rehabilitation: Preliminary findings with the bnave. Meurology Report (26), 72-78.

Witmer, B. G., \& Singer, M. J. (1998, June). Measuring presence in virtual environments: A presence questionnaire. Presence: Teleoper. Virtual Environ., 7(3), 225-240. Retrieved from http://dx.doi.org/10.1162/105474698565686 doi: 10.1162/105474698565686

Appendix

Result graphs 


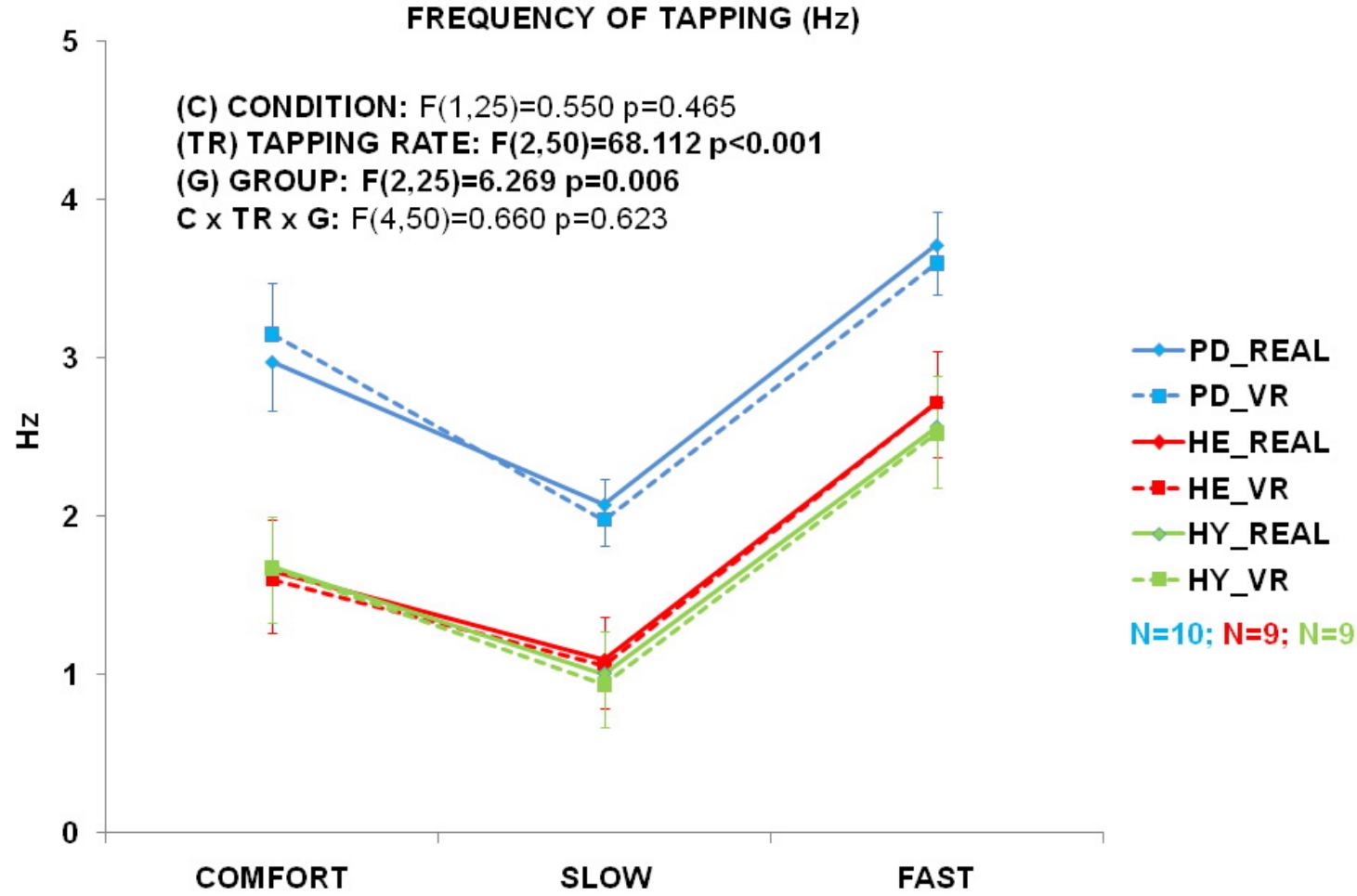

Figure A1. Figure illustrates the tapping frequency in the three groups, at the three tapping rates proposed (Comfort; Slow-Comfort; and Fast-Comfort), in both the Real (solid lines) and VR (dashed lines) environments. It is clear that the three groups modulated the tapping frequency, as slow was slower than comfort and comfort slower than fast. It is also clear than PD tapped faster than the other groups at all tapping rates (reflecting the typical hastening tendency in the disease) and proving validity, and this was observed in both environments (VR and Real execution overlapped) showing the lack of difference during virtual and real testing. 


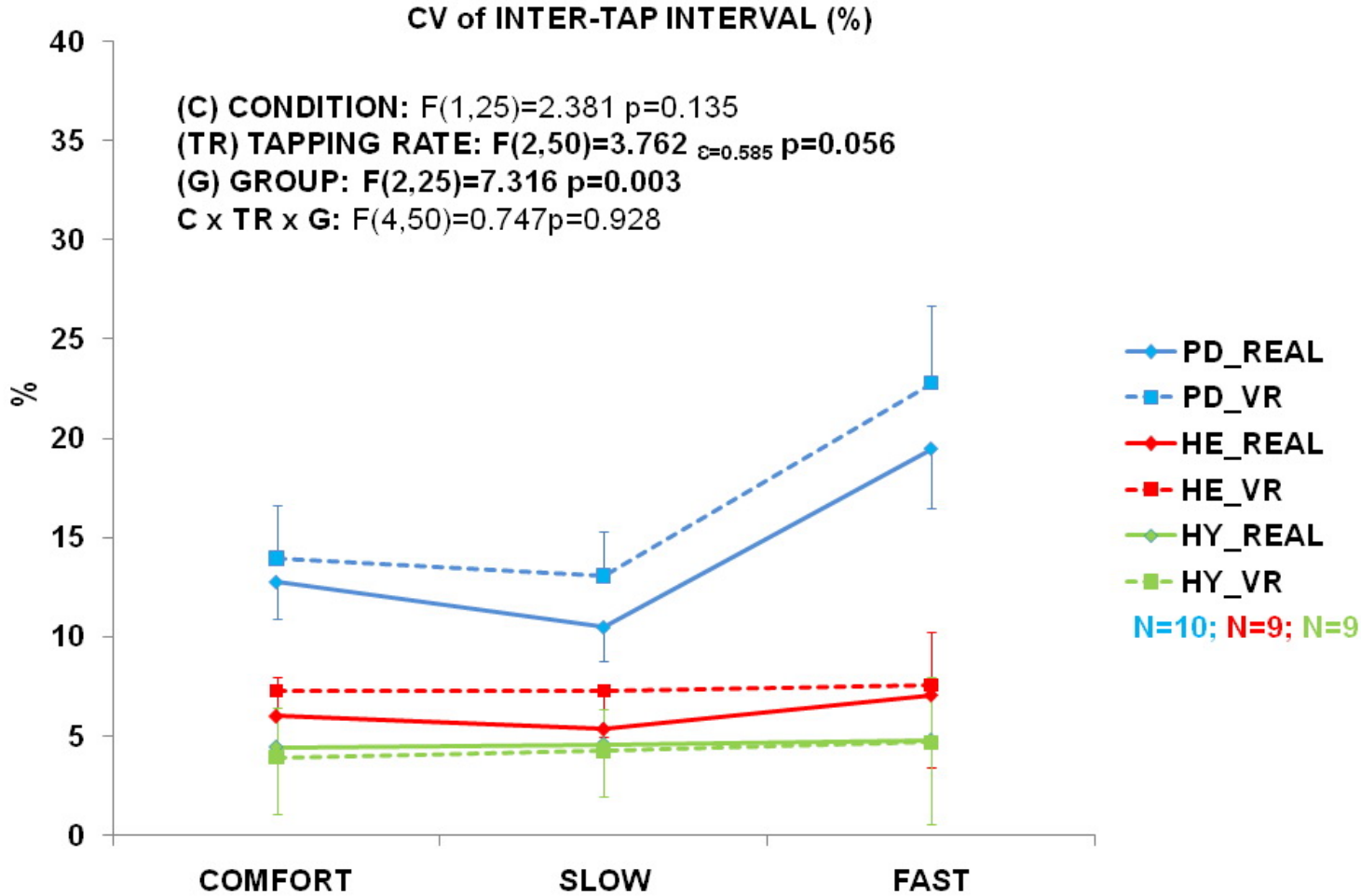

Figure A2. The plot represents the reliability analysis for the Frequency of execution while tapping. Taking all groups together a high level of consistency between execution in the VR and the Real environment is shown by the high ICC. The closer the points lay on the $45^{\circ}$ line the lesser the difference is between execution in both conditions; this is shown by the fact that the difference between execution in the VR minus Real was not significantly different from 0 - i.e. no difference. 


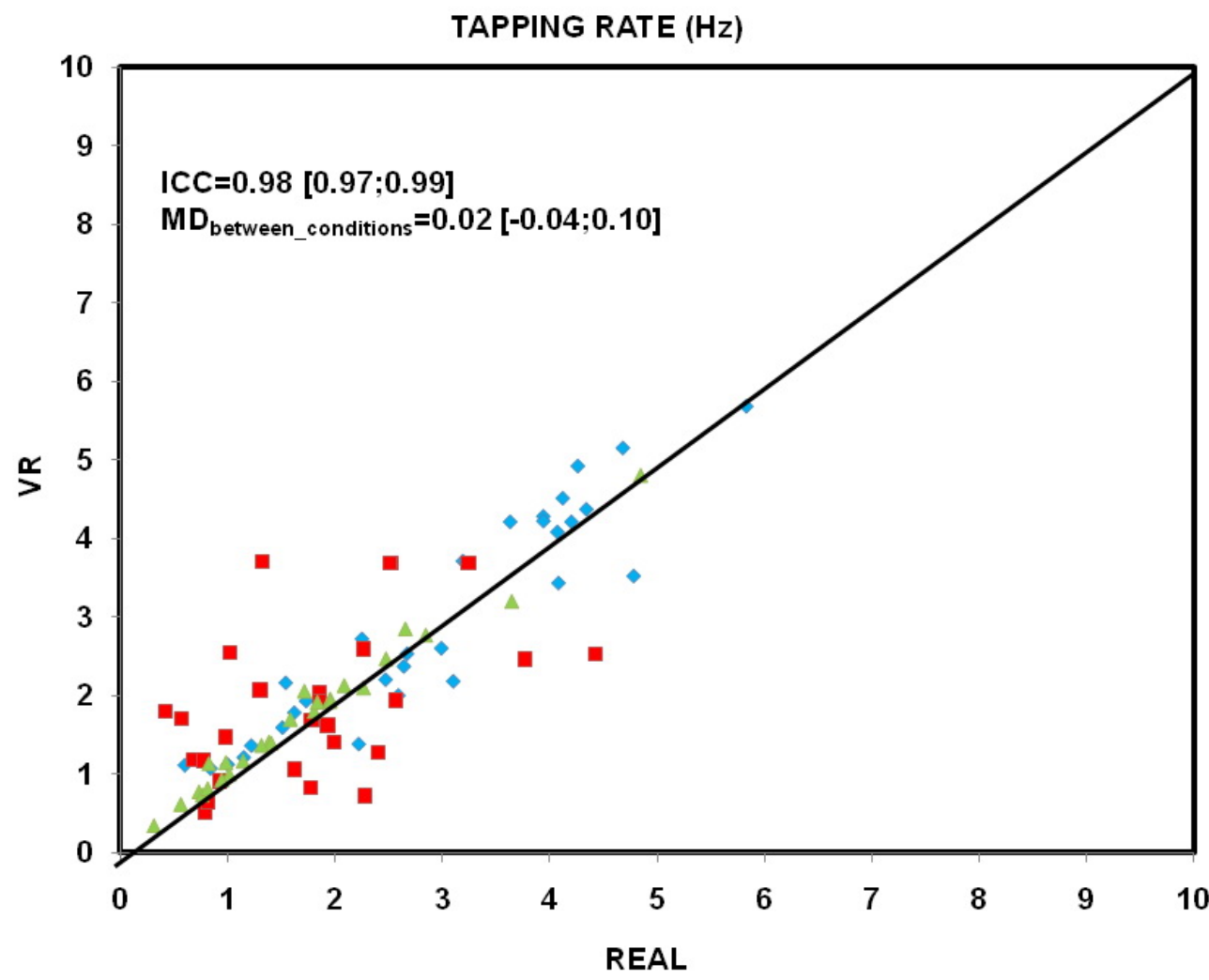

Figure A3. For the CV of inter-tap interval, execution in the VR and the Real environment did not present a different profile. Both conditions were able to differentiate the three groups. Clearly PD had larger variability that the other two groups, at all tapping rates, and HE had also slightly larger variability than the HY. There was a nearly significant trend to display larger variability at the fastest rate, but remarkably variability in the different rates was not differently assessed by VR or Real testing, and again showing validity of the VR to differentiate groups. 


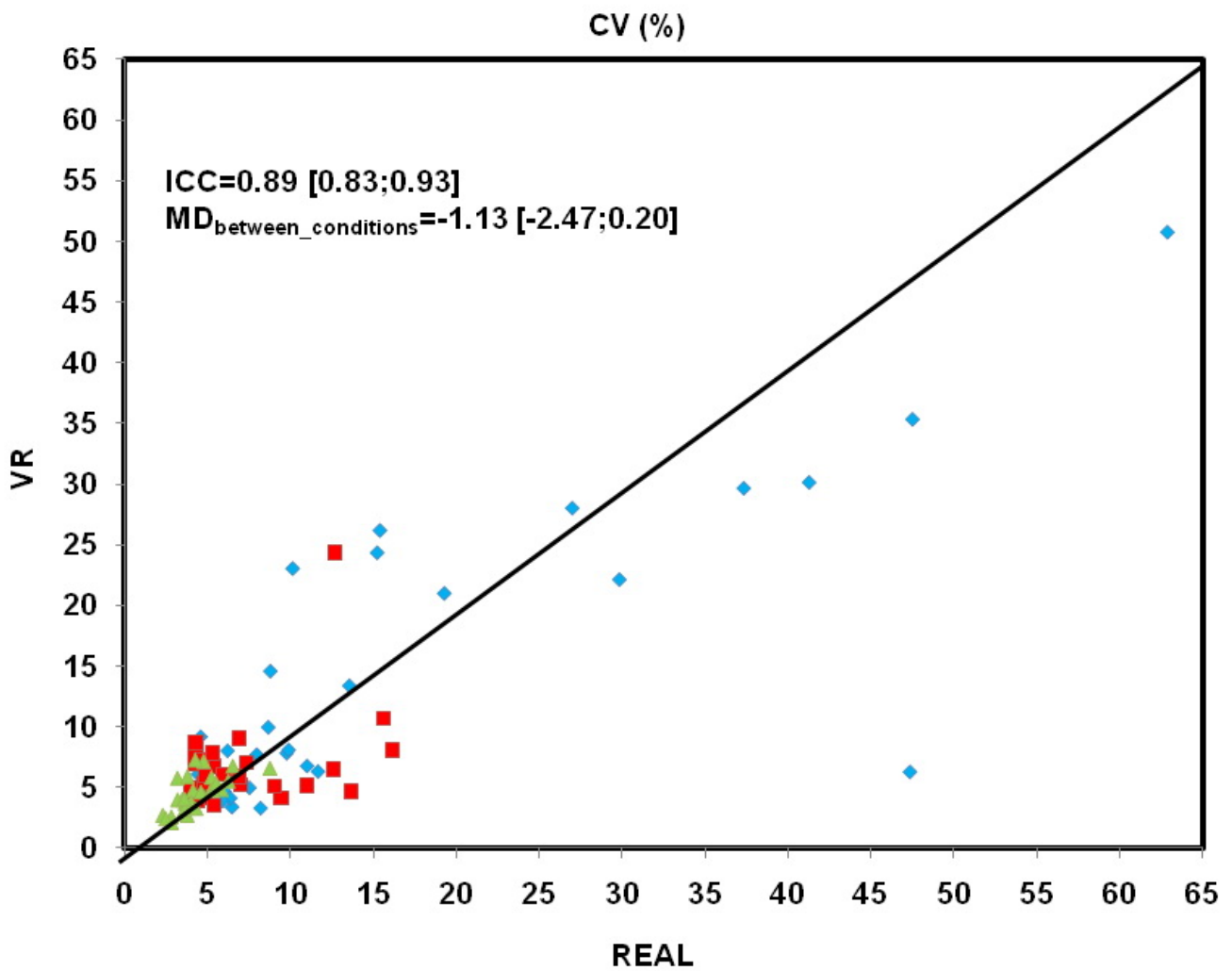

Figure A4. The figure shows, again, a great deal of consistency between Real and VR testing for the variable $\mathrm{CV}$ of inter-tap interval, $\mathrm{ICC}=0.89$. As mentioned before for the case of the frequency of execution, in the case of the CV, the mean difference between the execution in the VR and in the Real world was not significant different from 0 , showing the, overall, the suitability of VR testing in the evaluation of arrhythmikinesia. 
Frequency (hz) of each tap within a sequence in the three tapping rates in REAL and VR in a representative subject of each group
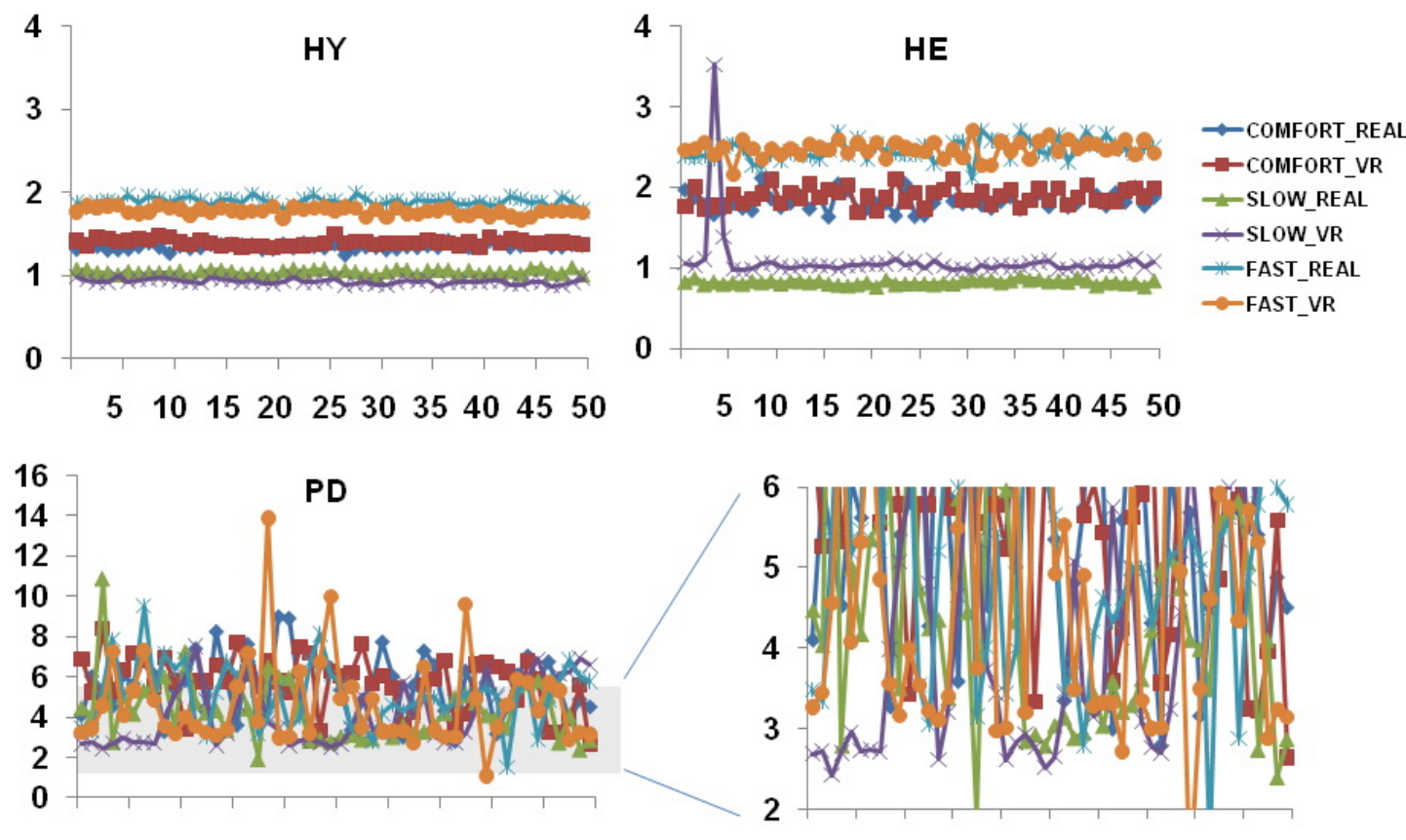

$\begin{array}{llllllllll}5 & 10 & 15 & 20 & 25 & 30 & 35 & 40 & 45 & 50\end{array}$

5101520253035404550

Figure A5. Representative behavior by one subject from each of the groups. It is clear the HY (a) presented the less variable pattern of tapping along the 50 taps sequence. The EC (b) presented a slightly larger variability and in some cases same hastening phenomenon (increase in tapping frequency) classic in aging. By large, PD (c) showed the larger variability, scaled to the same level of HY and EC in (d). In all cases the execution for VR and Real overlapped, regardless the tapping rate of execution and the kind of subject, advocating the use of VR as evaluation tool, as it does not distort execution from the obtained in Real-world testing. 